\title{
Kimyasal Gübrelerle Birlikte Uygulanan Vermikompost Kaynaklı Hümik Maddelerin Biberin Kısa Dönem Gelişimi ve Mineral Beslenmesine Etkisi
}

\author{
Cennet Yaylac1 $^{1 *}$, İbrahim Erdal ${ }^{2}$ \\ 1* Isparta Uygulamalı Bilimler Üniversitesi, Ziraat Fakültesi, Toprak Bilimi ve Bitki Besleme Bölümü, Isparta, (ORCID:0000-0002-0212-917X), \\ cyaylaci26@gmail.com \\ ${ }^{2}$ Isparta Uygulamalı Bilimler Üniversitesi, Ziraat Fakültesi, Toprak Bilimi ve Bitki Besleme Bölümü, Isparta, (ORCID: 0000-0001-8177-948X)
}

(İlk Geliş Tarihi 11 Kasım 2021 ve Kabul Tarihi 15 Aralık 2021)

(DOI: 10.31590/ejosat.1008881)

ATIF/REFERENCE: Yaylacı, C. \& Erdal, İ. (2021). Kimyasal Gübrelerle Birlikte Uygulanan Vermikompost Kaynaklı Hümik Maddelerin Biberin Kısa Dönem Gelişimi ve Mineral Beslenmesine Etkisi. Avrupa Bilim ve Teknoloji Dergisi, (31), 809-814.

Öz

Bu çalışmada vermikomposttan elde edilen hümik asit, fulvik asit ve hümik+fulvik asidin biberin gelişimi ve mineral beslenmesine etkisini incelemek amaçlanmıştır. Gübreli (300 ppm N, 200 ppm P, 200 ppm K, 5 ppm Zn, 10 ppm Fe) ve gübresiz koşullarda yürütülen denemede hümik maddelerin iki dozu (0 ve 1000 ppm) kullanılmıştır. Deneme sonunda bitkilerin kuru ağırlık değerleriyle $\mathrm{N}, \mathrm{P}, \mathrm{K}, \mathrm{Ca}, \mathrm{Mg}, \mathrm{Fe}, \mathrm{Cu}, \mathrm{Zn}$ ve $\mathrm{Mn}$ konsantrasyonları belirlenmiştir. Elde edilen sonuçlara göre hümik madde ve kimyasal gübre uygulamalarının bitkinin kuru ağırlığına anlamlı bir etkisi olmamıştır. Fakat hümik madde uygulamaları biber bitkisinin mineral beslenmesi üzerine olumlu etkiler göstermiştir.

Anahtar kelimeler: Humik maddeler, besin elementi alımı, bitki gelişimi

\section{The Effect of Combined Application of Vermicompost-Derived Humic Substances and Chemical Fertilizers on Short Time Growth and Mineral Nutrition of Pepper}

\begin{abstract}
Humic acid, fulvic acid and humic+fulvic acid obtained from vermicompost were used in the study to examine the effect of humic substances on the growth and mineral nutrition of pepper plants. Two levels ( 0 and $1000 \mathrm{ppm})$ of humic substances were applied with and without chemical fertilizers. After plants were harvested, dry weight and $\mathrm{N}, \mathrm{P}, \mathrm{K}, \mathrm{Ca}, \mathrm{Mg}, \mathrm{Fe}, \mathrm{Cu}, \mathrm{Zn}$ and $\mathrm{Mn}$ concentrations were determined. According to the results obtained, humikc substances and chemical fertilizer applications did not have a significant effect on the dry weight of the plant. However, humic substances applications showed positive effects on mineral nutrition of pepper plant.
\end{abstract}

Keywords: humic substances, nutrient uptake, plant growth 


\section{Giriş}

Geleneksel tarımdaki bilinçsiz gübre kullanımı çevresel bozulmalara neden olmakta, uzun vadede toprak verimliliğini olumsuz yönde etkileyebilmektedir. Sentetik gübrelerin toprağa olan zararını minimize etmenin en etkili yolu, toprak verimliliğinin organik bileşiklerle artırılmasıdır. $\mathrm{Bu}$ yönden toprak organik maddesi, tarımsal anlamda oldukça önem arz etmektedir (Halpern ve ark., 2015). Toprakta sürekli bir ayrışma içerisinde olan organik madde, toprağın mevcut su ve besin maddelerini koruyarak bitki gelişimini desteklemektedir (Lehmann ve ark., 2015). Toprak organik maddesi; strüktür oluşumuna katkı sağlamak, su tutma ve havalanma kapasitesini düzenlemek, yüzeyde kabuk tabakasının oluşumunu azaltmak gibi faydalarıyla toprak fiziksel özelliklerini iyileştirmekte ve bitki gelişimine iyi bir ortam hazırlamaktadır. Bunların yanında bitki besin elementi miktarlarını arttırmak, tuzluluğa ve $\mathrm{pH}$ değişimlerine karşı tamponlama kapasitesini geliştirmek, tarım ilaçları ve ağır metaller gibi birçok kirleticinin olumsuz etkilerini azaltmak, mikroorganizmalara karbon ve enerji kaynağı olmak gibi birçok yönden fayda sağlamaktadır (Taban ve ark., 2013). Organik maddenin büyük bir kısmını ise hümik maddeler oluşturmaktadır. Geniş bir $\mathrm{pH}$ aralığında tampon özelliği gösteren hümik maddelerin, toprakta katyon değişim kapasitesi oldukça yüksektir. Bu sayede metaller, hümik asitler ile şelat oluşturabilmektedir. Şelatlama ile de besin elementlerinin kaybı önlenerek, alına bilirliği arttırılmaktadır (Akıncı, 2011; Ünver ve özbek, 2011; Erdal ve ark., 2014). Hümik asitler; tohum, kök sistemi ve üst aksamı uyarabilmekte olup, bu sayede verimin artmasında önemli bir etken haline gelmektedir. Hümik asit içeren gübreler özellikle organik madde içeriği düşük topraklarda daha etkili olmakla birlikte doku besin dengesi, besinin bitkide transferi, kök gelişimi gibi birçok konuda olumlu sonuçlar meydana getirmektedir (Arduç, 2016). Hümik aside göre daha küçük moleküler yapıda olan fulvik asit, kolayca parçalanır. $\mathrm{Bu}$ yüzden hümik asit kadar kalıcı olmamaktadır. Hümik asit, yavaş parçalanarak toprakta daha uzun süre kalması nedeniyle toprak organik maddesini artırmak için fulvik asitlere göre daha çok tercih edilmektedir (Erol, 2011). Hümik maddelerin, bitkilerin mineral beslenmesi ve gelişimi üzerindeki olumlu etkileri yapılan birçok çalışma ile ortaya konmuştur (Atiyeh ve ark., 2002; Erdal ve ark., 2014; Canellas ve ark., 2015 ). Yapılan bu çalışmanın amacı hümik madde uygulamalarının erken dönemdeki biber bitkisinin mineral beslenmesine olan etkisini incelemektir.

\section{Materyal ve Yöntem}

Belirlenen ağırlıklardan yararlanarak vermikompostun HA, FA ve HFA oranları hesaplanmıştır. Bu hesaba göre, $184 \mathrm{~g}$ hümin olmayan madde elde edilmiştir. Çözünen kısım yani HFA $16 \mathrm{~g}$ bulunurken, asit ilavesinin ardından çöken koyu renkli kısım (HA) $8 \mathrm{~g}$ bulunmuştur.

Daha önce yapılan çalışmalara benzer olarak; toplam hümik+fulvik asit oran1 $\% 8$, toplam hümik asit $\% 4$ ve toplam fulvik asit \% 4 bulunmuştur (Dobbss ve ark., 2010; Canellas ve ark., 2013; Hernandez ve ark., 2015).

\section{Bulgular ve Tartışma}

Çizelge 1'de bitki kuru ağırlıkları ve $\mathrm{N}, \mathrm{P}, \mathrm{K}, \mathrm{Ca}$ ve $\mathrm{Mg}$ konsantrasyonları verilmiştir. Çalışmada hümik madde ve kimyasal gübre uygulamaları bahsi geçen besin elementleri
Deneme Isparta Uygulamalı Bilimler Üniversitesi, Ziraat Fakültesi, Toprak Bilimi ve Bitki Besleme Bölümü serasında, 3 tekerrürlü saksı denemesi olarak yürütülmüştür. Hava kuru hale getirilen topraklar 4 mm' lik elekten elenmiştir. İki kilogramlık saksılar ile yürütülen deneme 3 humik madde (HM) kaynağı (humik asit: HA, fulvik asit: FA ve humik+fulvik asit: HFA) iki HM dozu (D0:0 ve D1:1000 ppm), iki kimyasal gübre (KG) uygulaması (KG0: kontrol ve KG1: 300 ppm N, 200 ppm P, 200 ppm K, 5 ppm Zn, 10 ppm Fe) ve 3 paralelden oluşmaktadır. Gübre kaynakları olarak amonyum sülfat, TSP, potasyum sülfat, çinko sülfat ve demir sülfatın kullanıldığ çalışmada gübreleme, hazırlanan stok çözeltilerden her bir saksıya çözelti halinde verilerek gerçekleştirilmiştir. Dikim öncesinde yapılan HM ve gübre uygulamaları sonrasında topraklar iyice karıştırılmış ve saksılara doldurulduktan sonra fide dikimi yapılmıştır. Test bitkisi olarak biberin kullanıldığı çalışmada, dikimden itibaren 45 günlük sürenin sonunda bitkiler toprak yüzeyinden kesilerek hasat edilmiştir. Hasat edilen bitki örnekleri $65^{\circ} \mathrm{C}$ 'de sabit ağırlığa gelinceye kadar kurutulmuş ve kuru ağırlıkları belirlenmiştir. Daha sonra öğ̈̈ülen bu bitki örneklerinde $\mathrm{N}, \mathrm{P}$, $\mathrm{K}, \mathrm{Ca}, \mathrm{Mg}, \mathrm{Fe}, \mathrm{Cu}, \mathrm{Zn}$ ve $\mathrm{Mn}$ analizleri yapılmıştır. Elde edilen sonuçlar Tukey Çoklu Karşılaştırma Testi yapılarak, uygulamalar arası farklar istatistiksel olarak değerlendirilmiştir.

Çalışmada kullanılan toprak; Isparta Uygulamalı Bilimler Üniversitesi, Ziraat Fakültesi uygulama alanından alınmış olup, orta bünyeli, hafif alkali, kireçli, organik madde içeriği düşük bir topraktır.

Deneme için gerekli kullanılan hümik maddeler laboratuar koşullarında vermikomposttan üretilmiştir. Hümik ve fulvik asit fraksiyonlarının ayrıştırma işlemi, Rice (2008) tarafından bildirilen yöntem kullanılarak yapılmıştır. $\mathrm{Bu}$ yöntem için 2 litrelik erlende \% 2'lik $\mathrm{KOH}$ hazırlanarak içine firın kuru olarak $200 \mathrm{~g}$ vermikompost eklenmiştir. Hot plate üzerinde 70-75 ${ }^{\circ} \mathrm{C}$ 'de 7 saat karıştırılmıştır. Soğuduktan sonra hümin olmayan katı madde süzülerek etüvde kurutulmuş ve hümik ve fulvik asit içermeyen katı madde miktarı $184 \mathrm{~g}$ olarak belirlenmiştir. Süzülen ekstraktta ise HFA kalmıştır. Hümik ve fulvik asiti birbirinden ayırmak için ekstraktın pH'sı konsantre HCL ile 2'ye düşürülerek asitleştirilmiştir. Bu sayede hem asidik hem de bazik ortamda çözünebilen fulvik asit çözünmüş halde kalırken, sadece bazik ortamlarda çözünebilen hümik asit dibe çökelmiştir. Dibe çökelme işleminden sonra süspansiyon santrifüj edilerek fulvik asit hümik asitten ayrılmıştır. Fulvik asit alındıktan sonra kalan katı hümik asit etüvde kurutulmuş ve ağırlığı belirlenmiştir. Katı hümik asidi çözmek için ayrıca \% 1'lik $\mathrm{KOH}$ çözeltisi hazırlanmış ve katı hümik asit çözülmüştür.

yönünden farklı derecelerde istatistiksel olarak önemli bir farklılıklara sebep olmuştur. Kuru ağırlıklar arasında ise önemli bir fark görülmemiştir. Çalışmada en yüksek bitki kuru ağırlığ $(1,23 \mathrm{~g})$ temel gübreleme yapılan ve hümik madde uygulanmayan konulardan alınmıştır. Kuru ağırlık yönünden uygulamaların benzer sonuçlar vermesi, hasadın erken dönemde yapılması ile ilgili olabilir. Bitkilerin vejetatif aksamının yeterince gelişmemesi, uygulamalar arası verim yönünden benzer sonuçlara neden olmuş olabilir.

Çizelgede görüldüğü üzere uygulamalar arası farklar her element için değişiklik göstermiştir. Fosfor ve potasyum için uygulamalar arası farklar daha belirgin iken, kalsiyum ve magnezyum için genel interaksiyon ve gübre $\mathrm{x}$ hümik madde interaksiyonunda uygulamalar arası fark görülmemiştir. 
European Journal of Science and Technology

Çizelge 1: Uygulamaların biberin kuru ağırlığı (g/ bitki) ve N, P, K, Ca ve Mg konsantrasyonları (\%) üzerine etkisi

\begin{tabular}{|c|c|c|c|c|c|c|c|}
\hline \multicolumn{2}{|c|}{ Uygulamalar } & KA & $\mathrm{N}$ & $\mathrm{P}$ & $\mathrm{K}$ & $\mathrm{Ca}$ & $\mathrm{Mg}$ \\
\hline \multirow{12}{*}{$\begin{array}{l}\mathrm{KG} \times \mathrm{HM} \\
\times \mathrm{D}\end{array}$} & $\mathrm{KG}_{0} \mathrm{HA} \mathrm{D}_{0}$ & 1,17 & $2,78 \underline{\mathrm{d}^{*}}$ & $0,16 \underline{\mathrm{c}^{* *}}$ & $3,18 \underline{\mathrm{c}^{*}}$ & $2,80 \underline{\mathrm{d}^{*}}$ & 0,62 \\
\hline & $\mathrm{KG}_{0} \mathrm{HA} \mathrm{D_{1 }}$ & 1,10 & $2,78 \underline{\mathrm{d}}$ & $0,18 \underline{b c}$ & $3,36 \underline{\mathrm{c}}$ & $4,23 \underline{b}$ & 0,57 \\
\hline & $\mathrm{KG}_{0} \mathrm{FA} \mathrm{D}_{0}$ & 1,17 & $52,78 \underline{\mathrm{d}}$ & $0,16 \underline{\mathrm{c}}$ & $3,18 \underline{\mathrm{c}}$ & $2,80 \underline{\mathrm{d}}$ & 0,62 \\
\hline & $\mathrm{KG}_{0} \mathrm{FA} \mathrm{D}_{1}$ & 1,13 & $2,93 \underline{\mathrm{cd}}$ & $0,18 \underline{\mathrm{bc}}$ & $4,80 \underline{\bar{b}}$ & $3,00 \underline{\bar{d}}$ & 0,84 \\
\hline & $\mathrm{KG}_{0} \mathrm{HFA} \mathrm{D}_{0}$ & 1,17 & $2,78 \underline{\mathrm{d}}$ & $0,16 \underline{\mathrm{c}}$ & $3,18 \underline{\mathrm{c}}$ & $2,80 \underline{\underline{d}}$ & 0,62 \\
\hline & $\mathrm{KG}_{0} \mathrm{HFA} \mathrm{D}_{1}$ & 1,20 & $2,48 \underline{\mathrm{d}}$ & $0,16 \underline{\mathrm{c}}$ & $4,85 \underline{b}$ & $2,60 \underline{\mathrm{d}}$ & 0,65 \\
\hline & $\mathrm{KG}_{1} \mathrm{HA} \mathrm{D}_{0}$ & 1,23 & $3,90 \underline{a b}$ & $0,17 \underline{\mathrm{bc}}$ & $2,90 \underline{\mathrm{c}}$ & $3,73 \underline{\mathrm{bcd}}$ & 0,94 \\
\hline & $\mathrm{KG}_{1} \mathrm{HA} \mathrm{D}_{1}$ & 1,10 & $4,44 \underline{\mathrm{a}}$ & $0,17 \underline{\mathrm{bc}}$ & $2,98 \underline{\mathrm{c}}$ & $4,13 \underline{\mathrm{bc}}$ & 0,76 \\
\hline & $\mathrm{KG}_{1} \mathrm{FA} \mathrm{D}_{0}$ & 1,23 & $3,66 \underline{b}$ & $0,17 \underline{\mathrm{bc}}$ & $2,90 \underline{\mathrm{c}}$ & $3,73 \underline{\mathrm{bcd}}$ & 0,94 \\
\hline & $\mathrm{KG}_{1} \mathrm{FA} \mathrm{D}_{1}$ & 1,00 & $3,81 \underline{\mathrm{ab}}$ & $0,20 \underline{b}$ & $5,59 \underline{\mathrm{ab}}$ & $5,68 \underline{\mathrm{a}}$ & 1,00 \\
\hline & $\mathrm{KG}_{1} \mathrm{HFA} \mathrm{D}_{0}$ & 1,23 & $3,90 \underline{\mathrm{ab}}$ & $0,17 \underline{\mathrm{bc}}$ & $2,90 \underline{\mathrm{c}}$ & $3,73 \mathrm{bcd}$ & 0,94 \\
\hline & $\mathrm{KG}_{1} \mathrm{HFA} \mathrm{D}_{1}$ & 1,17 & $3,44 \underline{\mathrm{bc}}$ & $0,23 \underline{\mathrm{a}}$ & $6,03 \underline{\mathrm{a}}$ & $4,77 \underline{\mathrm{ab}}$ & 1,04 \\
\hline \multirow{6}{*}{$\mathrm{KG} \times \mathrm{HM}$} & $\mathrm{KG}_{0} \mathrm{HA}$ & 1,13 & $2,77 \mathbf{c}^{*}$ & $0,17 \mathbf{b c} * *$ & $3,27 \mathbf{b} *$ & $2,90 \mathbf{c d}^{*}$ & 0,60 \\
\hline & $\mathrm{KG}_{0} \mathrm{FA}$ & 1,15 & $2,86 \mathbf{c}$ & $0,17 \mathbf{b c}$ & $3,99 \mathbf{a}$ & 3,52 bc & 0,73 \\
\hline & $\mathrm{KG}_{0} \mathrm{HFA}$ & 1,18 & $2,63 \mathrm{c}$ & $0,16 \mathbf{c}$ & $4,01 \mathbf{a}$ & $2,70 \mathbf{e}$ & 0,64 \\
\hline & $\mathrm{KG}_{1} \mathrm{HA}$ & 1,17 & $4,17 \mathbf{a}$ & $0,17 \mathbf{b c}$ & $2,94 \mathbf{b}$ & $3,93 \mathbf{b}$ & 0,85 \\
\hline & $\mathrm{KG}_{1} \mathrm{FA}$ & 1,11 & $3,73 \mathbf{b}$ & $0,18 \mathbf{a b}$ & $4,25 \mathbf{a}$ & $4,71 \mathbf{a}$ & 0,97 \\
\hline & $\mathrm{KG}_{1} \mathrm{HFA}$ & 1,20 & $3,67 \mathbf{b}$ & $0,20 \mathbf{a}$ & $4,47 \mathbf{a}$ & $4,25 \mathbf{a b}$ & 0,99 \\
\hline \multirow{4}{*}{$\mathrm{KG} \times \mathrm{D}$} & $\mathrm{KG}_{0} \mathrm{D}_{0}$ & 1,17 & $2,78 B^{*}$ & 0,16 & $3,18 C^{* *}$ & $2,80 C^{*}$ & 0,62 \\
\hline & $\mathrm{KG}_{0} \mathrm{D}_{1}$ & 1,14 & $2,73 B$ & 0,18 & $4,33 B$ & $3,28 B C$ & 0,69 \\
\hline & $\mathrm{KG}_{1} \mathrm{D}_{0}$ & 1,23 & $3,82 \mathrm{~A}$ & 0,17 & $2,90 C$ & $3,73 B$ & 0,94 \\
\hline & $\mathrm{KG}_{1} \mathrm{D}_{1}$ & 1,09 & $3,90 \mathrm{~A}$ & 0,20 & $4,87 A$ & $4,86 A$ & 0,94 \\
\hline \multirow{6}{*}{$\mathrm{D} \times \mathrm{HM}$} & $\mathrm{HA} \mathrm{D}_{0}$ & 1,20 & $3,34 a b^{* *}$ & 0,16 & $3,04 b^{* *}$ & $3,27 b^{* *}$ & $0,78 a b^{*}$ \\
\hline & $\mathrm{HA} \mathrm{D}_{1}$ & 1,10 & $3,61 a$ & 0,18 & $3,17 b$ & $3,57 b$ & $0,67 b$ \\
\hline & $\mathrm{FA} \mathrm{D}_{0}$ & 1,20 & $3,22 a b$ & 0,16 & $3,04 b$ & $3,27 b$ & $0,78 a b$ \\
\hline & $\mathrm{FA} \mathrm{D}_{1}$ & 1,07 & $3,37 a$ & 0,19 & $5,19 a$ & $4,96 a$ & $0,92 a$ \\
\hline & $\mathrm{HFA} \mathrm{D}_{0}$ & 1,20 & $3,34 a b$ & 0,16 & $3,04 b$ & $3,27 b$ & $0,78 a b$ \\
\hline & $\mathrm{HFA} \mathrm{D}_{1}$ & 1,18 & $2,96 b$ & 0,20 & $5,44 a$ & $3,68 b$ & $0,85 a$ \\
\hline \multirow{2}{*}{$\mathrm{KG}$} & $\mathrm{KG}_{0}$ & 1,16 & $2,75 \mathbf{B} * *$ & $0,17 \mathbf{B} * *$ & 3,76 & $3,04 \mathbf{B} * *$ & $0,65 \mathbf{B} * *$ \\
\hline & $\mathrm{KG}_{1}$ & 1,16 & $3,86 \mathbf{A}$ & $0,18 \mathbf{A}$ & 3,88 & $4,30 \mathbf{A}$ & $0,94 \mathbf{A}$ \\
\hline \multirow{2}{*}{ D } & 0 & 1,20 & 3,30 & $0,16 b^{* *}$ & $3,04 b^{* *}$ & $3,27 b^{* *}$ & 0,78 \\
\hline & 1 & 1,12 & 3,31 & $0,19 \mathrm{a}$ & $4,60 \mathrm{a}$ & $4,07 \mathrm{a}$ & 0,81 \\
\hline \multirow{3}{*}{ HM } & HA & 1,15 & $3,47 \mathrm{~A}^{* *}$ & 0,17 & $3,11 \mathrm{~B} * *$ & $3,42 \mathrm{~B} * *$ & $0,72 \mathrm{~B}^{*}$ \\
\hline & FA & 1,13 & $3,30 \mathrm{AB}$ & 0,18 & $4,12 \mathrm{~A}$ & $4,11 \mathrm{~A}$ & $0,85 \mathrm{~A}$ \\
\hline & HFA & 1,19 & $3,15 \mathrm{~B}$ & 0,18 & $4,24 \mathrm{~A}$ & $3,48 \mathrm{~B}$ & $0,81 \mathrm{AB}$ \\
\hline
\end{tabular}

$*: \mathrm{p} \leq 0,05 \quad * *: \mathrm{p} \leq 0,01$

Çizelge 2'de biber bitkilerinin $\mathrm{Fe}, \mathrm{Cu}, \mathrm{Zn}, \mathrm{Mn}$ konsantrasyonları verilmiştir. Çizelgenin incelenmesinden de anlaşılacağı gibi yapılan uygulamaların hepsi, bahsi geçen elementler yönünden farklılığa sebep olmuştur. Özellikle genel interaksiyona bakıldığında kontrol saksılarına göre \% 95 artış göstererek Fe içeriğinin 595 ppm' e ulaştığı görülmektedir. Çinko için de yine önemli bir artış göstererek 50,5 ppm' e ulaştığı görülmektedir. Demir ve çinko yönünden en yüksek sonuç HFA uygulamalarından alınırken, bakır ve manganda en yüksek sonuçlar FA uygulamalarından alınmıştır.

Çizelge 2: Uygulamaların biberde $\mathrm{Fe}, \mathrm{Cu}, \mathrm{Zn}$ ve $\mathrm{Mn}$ konsantrasyonları üzerine etkisi (ppm)

\begin{tabular}{|c|c|c|c|c|c|}
\hline Uygulamalar & & $\mathrm{Fe}$ & $\mathrm{Cu}$ & $\mathrm{Zn}$ & Mn \\
\hline \multirow{12}{*}{$\begin{array}{l}\mathrm{KG} \times \mathrm{HM} \\
\times \mathrm{D}\end{array}$} & $\mathrm{KG}_{0} \mathrm{HA} \mathrm{D} \mathrm{D}_{0}$ & $305 \underline{c}$ & $10,6 \mathrm{e}^{* *}$ & $17,5 \mathrm{c}^{* *}$ & $139 \underline{\mathrm{bc}}$ \\
\hline & $\mathrm{KG}_{0} \mathrm{HA} \mathrm{D}_{1}$ & $448 \underline{\mathrm{abc}}$ & $12,8 \underline{\mathrm{de}}$ & $24,5 \underline{\mathrm{c}}$ & $103 \underline{\mathrm{c}}$ \\
\hline & $\mathrm{KG}_{0} \mathrm{FA} \mathrm{D}_{0}$ & $305 \underline{c}$ & $10,6 \underline{\mathrm{e}}$ & $17,5 \underline{\mathrm{c}}$ & $139 \underline{\mathrm{bc}}$ \\
\hline & $\mathrm{KG}_{0} \mathrm{FA} \mathrm{D}_{1}$ & $398 \underline{b c}$ & 14,0 cde & $23,8 \underline{\mathrm{c}}$ & $178 \underline{\mathrm{ab}}$ \\
\hline & $\mathrm{KG}_{0} \mathrm{HFA} \mathrm{D}_{0}$ & $305 \overline{\mathrm{c}}$ & $10,6 \underline{\mathrm{e}}$ & $17,5 \underline{\mathrm{c}}$ & $139 \underline{\mathrm{bc}}$ \\
\hline & $\mathrm{KG}_{0} \mathrm{HFA} \mathrm{D}_{1}$ & $403 \underline{\mathrm{b}} \underline{\mathrm{c}}$ & $24,1 \underline{\mathrm{ab}}$ & $19,8 \underline{\mathrm{c}}$ & $157 \underline{\mathrm{bc}}$ \\
\hline & $\mathrm{KG}_{1} \mathrm{HA} \mathrm{D}_{0}$ & $520 \overline{\mathrm{ab}}$ & $14,7 \overline{\mathrm{cde}}$ & $36,4 \underline{\bar{b}}$ & $128 \underline{\mathrm{bc}}$ \\
\hline & $\mathrm{KG}_{1} \mathrm{HA} \mathrm{D_{1 }}$ & $499 \underline{\overline{a b}}$ & $16,7 \underline{\mathrm{cd}}$ & $34,8 \underline{\bar{b}}$ & $163 \underline{\mathrm{bc}}$ \\
\hline & $\mathrm{KG}_{1} \mathrm{FA} \mathrm{D}_{0}$ & $520 \underline{\mathrm{ab}}$ & 14,7 cde & $36,4 \underline{b}$ & $128 \underline{\mathrm{bc}}$ \\
\hline & $\mathrm{KG}_{1} \mathrm{FA} \mathrm{D}_{1}$ & $525 \underline{\underline{\mathrm{ab}}}$ & $26,0 \underline{\mathrm{a}}$ & $49,6 \underline{\mathrm{a}}$ & $247 \underline{\mathrm{a}}$ \\
\hline & $\mathrm{KG}_{1} \mathrm{HFA} \mathrm{D}_{0}$ & $520 \underline{\overline{\mathrm{ab}}}$ & 14,7 cde & $36,4 \underline{\bar{b}}$ & $128 \underline{\mathrm{b} c}$ \\
\hline & $\mathrm{KG}_{1} \mathrm{HFA} \mathrm{D}_{1}$ & $595 \overline{\mathrm{a}}$ & $19,3 \overline{\mathrm{bc}}$ & $50,5 \overline{\mathrm{a}}$ & $198 \overline{\mathrm{ab}}$ \\
\hline $\mathrm{KG} \times \mathrm{HM}$ & $\mathrm{KG}_{0} \mathrm{HA}$ & $377 \mathbf{b}$ & $11,7 \mathbf{c} * *$ & $21,0 \mathrm{c} * *$ & $121 \mathbf{b}$ \\
\hline
\end{tabular}


Avrupa Bilim ve Teknoloji Dergisi

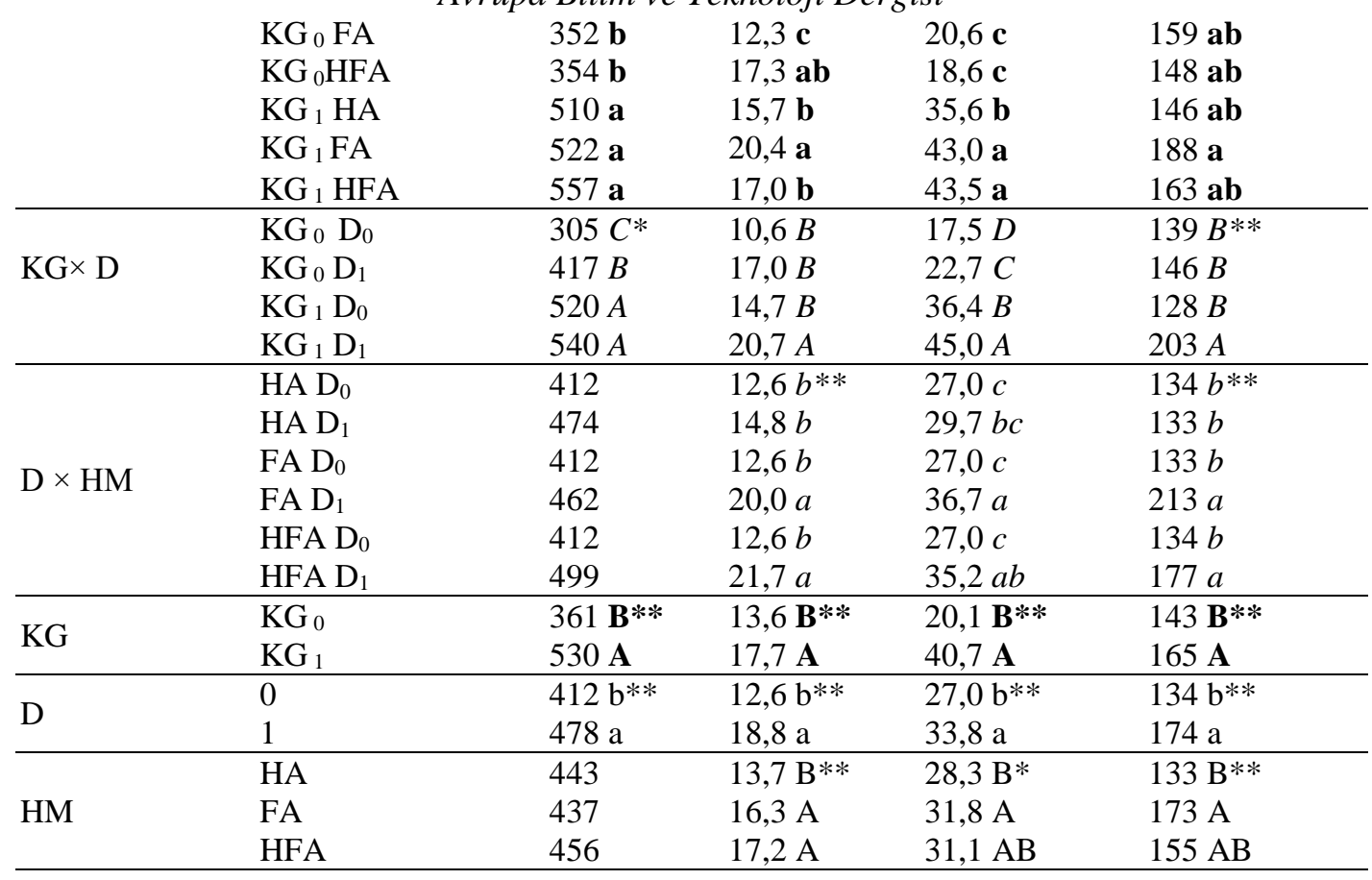

$*: \mathrm{p} \leq 0,05 \quad * *: \mathrm{p} \leq 0,01$

Çizelge 3' te bitki gelişimi boyunca saksıdan kaldırılan toplam $\mathrm{N}, \mathrm{P}, \mathrm{K}, \mathrm{Ca}$ ve $\mathrm{Mg}$ miktarları verilmiştir. Çizelge incelendiğinde farklı uygulamaların $\mathrm{P}$ dışındaki makro elementler için istatistiksel olarak önemli farklara neden olduğu görülmektedir. En fazla besin elementi kimyasal gübre ve HFA uygulaması yapılan saksılardan kaldırılmıştır.

Çizelge 3: Uygulamaların biberde N, P, K, Ca ve Mg alımı üzerine etkisi (mg/saksı)

\begin{tabular}{|c|c|c|c|c|c|c|}
\hline \multicolumn{2}{|c|}{ Uygulamalar } & \multirow{2}{*}{$\frac{\mathrm{N}}{3,25}$} & \multirow{2}{*}{$\frac{\mathrm{P}}{1,83}$} & \multirow{2}{*}{$\begin{array}{l}\mathrm{K} \\
37,1 \underline{\mathrm{c}}\end{array}$} & \multirow{2}{*}{$\begin{array}{l}\mathrm{Ca} \\
32,6 \underline{b c}\end{array}$} & \multirow{2}{*}{$\begin{array}{l}\mathrm{Mg} \\
7,23 \underline{\mathrm{bc}}\end{array}$} \\
\hline \multirow{12}{*}{$\begin{array}{l}\mathrm{KG} \times \mathrm{HM} \\
\times \mathrm{D}\end{array}$} & $\mathrm{KG}_{0} \mathrm{HA} \mathrm{D}_{0}$ & & & & & \\
\hline & $\mathrm{KG}_{0} \mathrm{HA} \mathrm{D_{1 }}$ & 3,05 & 2,01 & $37,1 \underline{\mathrm{c}}$ & $33,0 \underline{b c}$ & $6,36 \underline{\mathrm{c}}$ \\
\hline & $\mathrm{KG}_{0} \mathrm{FA} \mathrm{D}_{0}$ & 3,25 & 1,83 & $37,1 \underline{\mathrm{c}}$ & $32,6 \underline{b c}$ & $7,23 \underline{b c}$ \\
\hline & $\mathrm{KG}_{0} \mathrm{FA} \mathrm{D}_{1}$ & 3,33 & 2,04 & $54,2 \underline{\bar{b}}$ & $47,9 \overline{\mathrm{ab}}$ & $9,48 \overline{\mathrm{abc}}$ \\
\hline & $\mathrm{KG}_{0} \mathrm{HFA} \mathrm{D}_{0}$ & 3,25 & 1,83 & $37,1 \underline{\mathrm{c}}$ & $32,6 \overline{\mathrm{bc}}$ & $7,23 \overline{\mathrm{bc}}$ \\
\hline & $\mathrm{KG}_{0} \mathrm{HFA} \mathrm{D}_{1}$ & 2,97 & 1,98 & $58,1 \underline{\mathrm{ab}}$ & $31,2 \overline{\mathrm{c}}$ & $7,81 \overline{\mathrm{abc}}$ \\
\hline & $\mathrm{KG}_{1} \mathrm{HA} \mathrm{D}_{0}$ & 4,82 & 2,11 & $35,6 \underline{\mathrm{c}}$ & $45,3 \underline{a b c}$ & $11,4 \underline{\mathrm{ab}}$ \\
\hline & $\mathrm{KG}_{1} \mathrm{HA} \mathrm{D_{1 }}$ & 4,89 & 1,87 & $32,7 \underline{\mathrm{c}}$ & $45,8 \underline{\mathrm{abc}}$ & $8,39 \underline{\mathrm{abc}}$ \\
\hline & $\mathrm{KG}_{1} \mathrm{FA} \mathrm{D}_{0}$ & 4,54 & 2,11 & $35,6 \overline{\mathrm{c}}$ & $45,3 \overline{\mathrm{abc}}$ & $11,5 \overline{\mathrm{ab}}$ \\
\hline & $\mathrm{KG}_{1} \mathrm{FA} \mathrm{D}$ & 3,81 & 1,97 & $55,9 \underline{\mathrm{ab}}$ & $56,8 \underline{\mathrm{a}}$ & $10,0 \overline{\mathrm{abc}}$ \\
\hline & $\mathrm{KG}_{1} \mathrm{HFA} \mathrm{D}_{0}$ & 4,82 & 2,11 & $35,6 \overline{\mathrm{c}}$ & $45,3 \underline{\mathrm{abc}}$ & $11,5 \overline{\mathrm{ab}}$ \\
\hline & $\mathrm{KG}_{1} \mathrm{HFA} \mathrm{D}_{1}$ & 4,02 & 2,63 & $69,1 \overline{\mathrm{a}}$ & $54,7 \overline{\mathrm{a}}$ & $12,0 \overline{\mathrm{a}}$ \\
\hline \multirow{6}{*}{$\mathrm{KG} \times \mathrm{HM}$} & $\mathrm{KG}_{0} \mathrm{HA}$ & $3,15 \mathrm{c}^{*}$ & 1,92 & $37,1 \mathbf{b}$ & $32,8 \mathbf{c}$ & $6,80 \mathbf{d}$ \\
\hline & $\mathrm{KG}_{0} \mathrm{FA}$ & 3,29 bc & 1,94 & $45,6 \mathbf{a}$ & 40,3 bc & 8,36 bcd \\
\hline & $\mathrm{KG}_{0} \mathrm{HFA}$ & $3,11 \mathbf{c}$ & 1,90 & $47,6 \mathbf{a}$ & $31,9 \mathbf{c}$ & 7,52 cd \\
\hline & $\mathrm{KG}_{1} \mathrm{HA}$ & $4,86 \mathbf{a}$ & 1,99 & $34,1 \mathbf{b}$ & $45,6 \mathbf{a b}$ & 9,94 abc \\
\hline & $\mathrm{KG}_{1} \mathrm{FA}$ & $4,17 \mathbf{a b c}$ & 2,04 & $45,8 \mathbf{a}$ & $51,1 \mathbf{a}$ & $10,8 \mathbf{a b}$ \\
\hline & $\mathrm{KG}_{1} \mathrm{HFA}$ & 4,42 ab & 2,37 & $52,3 \mathbf{a}$ & $50,0 \mathbf{a}$ & $11,8 \mathbf{a}$ \\
\hline \multirow{4}{*}{$\mathrm{KG} \times \mathrm{D}$} & $\mathrm{KG}_{0} \mathrm{D}_{0}$ & $3,25 B^{*}$ & 1,83 & $37,1 B$ & $32,6 C$ & $7,23 B$ \\
\hline & $\mathrm{KG}_{0} \mathrm{D}_{1}$ & $3,12 B$ & 2,01 & $49,8 \mathrm{~A}$ & $37,4 C$ & $7,88 B$ \\
\hline & $\mathrm{KG}_{1} \mathrm{D}_{0}$ & $4,72 \mathrm{~A}$ & 2,11 & $35,6 B$ & $45,3 B$ & $11,5 \mathrm{~A}$ \\
\hline & $\mathrm{KG}_{1} \mathrm{D}_{1}$ & $4,24 \mathrm{~A}$ & 2,16 & $52,6 \mathrm{~A}$ & $52,4 A$ & $10,2 \mathrm{~A}$ \\
\hline \multirow{6}{*}{$\mathrm{D} \times \mathrm{HM}$} & $\mathrm{HA} \mathrm{D}_{0}$ & 4,03 & 1,97 & $36,3 c^{* *}$ & $39,0 b^{*}$ & 9,36 \\
\hline & $\mathrm{HA} \mathrm{D}_{1}$ & 3,97 & 1,94 & $34,9 c$ & $39,4 b$ & 7,38 \\
\hline & $\mathrm{FA} \mathrm{D}_{0}$ & 3,89 & 1,97 & $36,3 c$ & $39,0 \mathrm{~b}$ & 9,36 \\
\hline & $\mathrm{FA} \mathrm{D}_{1}$ & 3,57 & 2,01 & $55,1 b$ & $52,4 a$ & 9,75 \\
\hline & $\mathrm{HFA} \mathrm{D}_{0}$ & 4,03 & 1,97 & $36,3 c$ & $39,0 \mathrm{~b}$ & 9,36 \\
\hline & $\mathrm{HFA} \mathrm{D}_{1}$ & 3,49 & 2,30 & $63,6 a$ & $42,9 b$ & 9,93 \\
\hline \multirow{2}{*}{$\mathrm{KG}$} & $\mathrm{KG}_{0}$ & $3,18 \mathbf{B} * *$ & $1,92 \mathbf{B} *$ & 43,4 & $35,0 \mathbf{B} * *$ & $7,56 \mathbf{B} * *$ \\
\hline & $\mathrm{KG}_{1}$ & $4,48 \mathbf{A}$ & $2,13 \mathbf{A}$ & 44,1 & $48,9 \mathbf{A}$ & $10,8 \mathbf{A}$ \\
\hline
\end{tabular}


European Journal of Science and Technology

\begin{tabular}{lllllll}
\hline \multirow{2}{*}{$\mathrm{D}$} & 0 & 3,99 & 1,97 & $36,3 \mathrm{~b}^{* *}$ & $39,0 \mathrm{~b}^{* *}$ & 9,36 \\
& 1 & 3,68 & 2,08 & $51,2 \mathrm{a}$ & $44,9 \mathrm{a}$ & 9,02 \\
\hline \multirow{3}{*}{$\mathrm{HM}$} & $\mathrm{HA}$ & 4,00 & 1,96 & $35,6 \mathrm{~B} * *$ & $39,2 \mathrm{~B}^{*}$ & 8,37 \\
& FA & 3,73 & 1,99 & $45,7 \mathrm{~A}$ & $45,7 \mathrm{~A}$ & 9,56 \\
& HFA & 3,76 & 2,14 & $50,0 \mathrm{~A}$ & $41,0 \mathrm{AB}$ & 9,64 \\
\hline
\end{tabular}

$*: \mathrm{p} \leq 0,05 \quad * *: \mathrm{p} \leq 0,01$

Çizelge 4' te bitki gelişimi boyunca saksıdan kaldırılan toplam $\mathrm{Fe}, \mathrm{Cu}, \mathrm{Zn}$ ve $\mathrm{Mn}$ miktarları verilmiştir. Çizelge incelendiğinde her bir besin elementi için farklı uygulamaların istatistiksel olarak önemli farklara yol açtığı görülmektedir. Yine kimyasal gübre ve HFA uygulaması yapılan konulardan en fazla besin elementi kaldırılmıştır.

Çizelge 4: Uygulamaların biberde $\mathrm{Fe}, \mathrm{Cu}, \mathrm{Zn}$ ve $\mathrm{Mn}$ alımı üzerine etkisi ( $\mu \mathrm{g} / \mathrm{saksı}$ )

\begin{tabular}{|c|c|c|c|c|c|}
\hline Uygulamalar & & $\mathrm{Fe}$ & $\mathrm{Cu}$ & $\mathrm{Zn}$ & $\mathrm{Mn}$ \\
\hline \multirow{12}{*}{$\begin{array}{l}\mathrm{KG} \times \mathrm{HM} \\
\times \mathrm{D}\end{array}$} & $\mathrm{KG}_{0} \mathrm{HA} \mathrm{D}_{0}$ & 356 & $12,3 \underline{\mathrm{d}^{*}}$ & $20,4 \underline{\mathrm{c}}$ & $1713 \underline{\mathrm{c}}$ \\
\hline & $\mathrm{KG}_{0} \mathrm{HA} \mathrm{D_{1 }}$ & 495 & $14,1 \underline{\mathrm{cd}}$ & $26,9 \underline{\mathrm{bc}}$ & $1436 \underline{c}$ \\
\hline & $\mathrm{KG}_{0} \mathrm{FA} \mathrm{D}_{0}$ & 356 & $12,3 \underline{\mathrm{d}}$ & $20,4 \underline{\mathrm{c}}$ & $1713 \underline{\mathrm{c}}$ \\
\hline & $\mathrm{KG}_{0} \mathrm{FA} \mathrm{D}_{1}$ & 451 & $15,8 \underline{\mathrm{cd}}$ & $27,0 \underline{b c}$ & $2830 \underline{b c}$ \\
\hline & $\mathrm{KG}_{0} \mathrm{HFA} \mathrm{D}_{0}$ & 356 & $12,3 \underline{\mathrm{d}}$ & $20,4 \underline{\mathrm{c}}$ & $1713 \underline{\mathrm{c}}$ \\
\hline & $\mathrm{KG}_{0} \mathrm{HFA} \mathrm{D}_{1}$ & 484 & $28,9 \underline{\mathrm{a}}$ & $23,7 \underline{\mathrm{c}}$ & $4652 \underline{\mathrm{ab}}$ \\
\hline & $\mathrm{KG}_{1} \mathrm{HA} \mathrm{D}_{0}$ & 649 & $18,1 \underline{\mathrm{bcd}}$ & $45,0 \underline{\mathrm{ab}}$ & $2342 \underline{b c}$ \\
\hline & $\mathrm{KG}_{1} \mathrm{HA} \mathrm{D}_{1}$ & 546 & $18,5 \underline{\mathrm{bcd}}$ & $38,3 \underline{\mathrm{abc}}$ & $3024 \underline{b c}$ \\
\hline & $\mathrm{KG}_{1} \mathrm{FA} \mathrm{D}_{0}$ & 649 & $18,1 \mathrm{bcd}$ & $45,0 \underline{\mathrm{ab}}$ & $2342 \underline{b c}$ \\
\hline & $\mathrm{KG}_{1} \mathrm{FA} \mathrm{D}_{1}$ & 525 & $26,0 \underline{\mathrm{ab}}$ & $49,6 \underline{\mathrm{a}}$ & $6457 \underline{\mathrm{a}}$ \\
\hline & $\mathrm{KG}_{1} \mathrm{HFA} \mathrm{D}_{0}$ & 649 & $18,1 \underline{\mathrm{bcd}}$ & $45,0 \underline{\mathrm{ab}}$ & $2342 \underline{b c}$ \\
\hline & $\mathrm{KG}_{1} \mathrm{HFA} \mathrm{D}_{1}$ & 696 & $22,7 \underline{a b c}$ & $58,7 \underline{\mathrm{a}}$ & $4489 \underline{\mathrm{ab}}$ \\
\hline \multirow{6}{*}{$\mathrm{KG} \times \mathrm{HM}$} & $\mathrm{KG}_{0} \mathrm{HA}$ & $425 \mathrm{~b}$ & $13,2 \mathbf{b}^{*}$ & $23,7 \mathbf{b}$ & $1575 \mathrm{c}$ \\
\hline & $\mathrm{KG}_{0} \mathrm{FA}$ & $403 \mathbf{b}$ & $14,1 \mathbf{b}$ & $23,7 \mathbf{b}$ & 2271 bc \\
\hline & $\mathrm{KG}_{0} \mathrm{HFA}$ & $420 \mathbf{b}$ & $20,6 \mathbf{a}$ & $22,1 \mathbf{b}$ & 3182 abc \\
\hline & $\mathrm{KG}_{1} \mathrm{HA}$ & $598 \mathbf{a b}$ & $18,3 \mathbf{a b}$ & $41,6 \mathbf{a}$ & 2683 bc \\
\hline & $\mathrm{KG}_{1} \mathrm{FA}$ & $587 \mathbf{a b}$ & $22,1 \mathbf{a}$ & $47,3 \mathbf{a}$ & $4399 \mathbf{a}$ \\
\hline & $\mathrm{KG}_{1} \mathrm{HFA}$ & $673 \mathbf{a}$ & $20,4 \mathbf{a}$ & $51,8 \mathbf{a}$ & $3416 \mathbf{a b}$ \\
\hline \multirow{4}{*}{$\mathrm{KG} \times \mathrm{D}$} & $\mathrm{KG}_{0} \mathrm{D}_{0}$ & $356 C^{*}$ & $12,3 B$ & $20,4 B$ & $1713 C$ \\
\hline & $\mathrm{KG}_{0} \mathrm{D}_{1}$ & $477 B C$ & $19,6 A$ & $25,9 B$ & $2973 B$ \\
\hline & $\mathrm{KG}_{1} \mathrm{D}_{0}$ & $649 A$ & $18,1 A$ & $45,0 \mathrm{~A}$ & $2342 B C$ \\
\hline & $\mathrm{KG}_{1} \mathrm{D}_{1}$ & $589 A B$ & $22,4 A$ & $48,9 A$ & $4657 A$ \\
\hline \multirow{6}{*}{$\mathrm{D} \times \mathrm{HM}$} & $\mathrm{HA} \mathrm{D}_{0}$ & 503 & $15,2 b^{* *}$ & 32,7 & $2027 b^{* *}$ \\
\hline & $\mathrm{HA} \mathrm{D}_{1}$ & 521 & $16,3 b$ & 32,6 & $2230 b$ \\
\hline & FA D 0 & 503 & $15,2 b$ & 32,7 & $2027 b$ \\
\hline & $\mathrm{FA} \mathrm{D}_{1}$ & 488 & $20,9 a b$ & 38,3 & $4643 a$ \\
\hline & $\mathrm{HFA} \mathrm{D}_{0}$ & 503 & $15,2 b$ & 32,7 & $2027 b$ \\
\hline & $\mathrm{HFA} \mathrm{D}_{1}$ & 590 & $25,8 a$ & 41,2 & $4570 a$ \\
\hline \multirow{2}{*}{$\mathrm{KG}$} & $\mathrm{KG}_{0}$ & $416 \mathbf{B} * *$ & $16,0 \mathbf{B} * *$ & $23,2 \mathbf{B} * *$ & $2343 \mathbf{B} * *$ \\
\hline & $\mathrm{KG}_{1}$ & $619 \mathrm{~A}$ & $20,2 \mathbf{A}$ & $47,0 \mathbf{A}$ & 3499 A \\
\hline \multirow{2}{*}{ D } & 0 & 503 & $15,2 b^{* *}$ & 32,7 & $2027 b^{* *}$ \\
\hline & 1 & 533 & $21,0 \mathrm{a}$ & 37,4 & $3815 \mathrm{a}$ \\
\hline \multirow{3}{*}{ HM } & HA & 512 & $15,8 \mathrm{~B} * *$ & 32,7 & $2129 \mathrm{~B} * *$ \\
\hline & FA & 495 & $18,1 \mathrm{AB}$ & 35,5 & 3335 A \\
\hline & HFA & 546 & $20,5 \mathrm{~A}$ & 37,0 & 3299 A \\
\hline
\end{tabular}

$*: \mathrm{p} \leq 0,05 \quad * *: \mathrm{p} \leq 0,01$

Mineral gübrelerin bitki beslenmesi üzerine olan etkileri yapılan birçok çalışma ile ortaya konmuştur (Aliyu, 2000; López ve ark., 2011; Siebielec ve ark., 2018). Bu doğrultuda bitkilerin $\mathrm{K}$, $\mathrm{Fe}$ ve $\mathrm{Zn}$ içeriklerinde uygulamalar arası farklılıkların oluşması yapılan temel gübrelemeden kaynaklanmış olabilir. Sonuçlara genel olarak bakıldığında hümik madde uygulamasının bireysel etkilerinden, en yüksek sonuçların HFA uygulaması yapılan konulardan elde edildiği anlaşılmaktadır. $\mathrm{Bu}$ durumda hümik+fulvik asit uygulamasının, hümik asit ve fulvik asit'in bireysel olarak uygulanmasından daha verimli olacağ fikrine varilabilir.

Genel olarak sonuçlara bakıldığında hasat sonrası bitkilerin $\mathrm{Fe}, \mathrm{Cu}, \mathrm{Zn}$ ve $\mathrm{Mn}$ içerikleri yeterli düzeyde bulunmuştur. Yine makro elementlerden $\mathrm{K}$ ve $\mathrm{Mg}$ yeterli düzeyde bulunurken; $\mathrm{Ca}$ fazla, $\mathrm{P}$ ise yetersiz bulunmuştur. Bitkilerdeki bu $\mathrm{Ca}$ fazlalığı göz önüne alındığında; sulama suyundan gelen $\mathrm{CaCO} 3$ 'ın toprak mikroorganizmalarınca ayrıştırılması sonucu, toprak çözeltisinde Ca konsantrasyonunun artmış olması mümkündür (Okur ve ark., 2007; Abdulwahhab ve Șeker , 2020). Bunun yanında kullanılan 


\section{Avrupa Bilim ve Teknoloji Dergisi}

toprağın kireçli olması da, bu durumun nedenleri arasında sayılabilir. Fosfor içeriğinin yetersizliği ise; organik madde içeriği düşük olan bir toprak kullanıldığ 1 düşünüldüğünde, yapılan hümik madde ve kimyasal gübre uygulamalarının da etkisi görülmeden hasat edilmesine bağlı olabilir.

Sonuç olarak hümik madde uygulamalarının erken dönemdeki biber bitkisinin mineral beslenmesi üzerine olumlu etkileri olmuştur. Fakat bu etkileri daha detaylı bir şekilde incelemek için hümik maddeleri birkaç farklı dozda uygulamak daha uygun olacaktır.

\section{Kaynaklar}

Aliyu, L. (2000). Effect of organic and mineral fertilizers on growth, yield and composition of pepper (Capsicum annuum L.). Biological agriculture \& horticulture, 18(1), 29-36. https://doi.org/10.1080/01448765.2000.9754862

Abdulwahhab, Q., \& Şeker, C. Farklı Dozlarda Kireç Uygulamasının İki Farklı Tekstürdeki Toprağın Bazı Fiziksel Ve Kimyasal Özellikleri Üzerine Etkisi. Toprak Su Dergisi, $9(2), \quad 80-87$. https://doi.org/10.21657/topraksu.699821

Atiyeh, R. M., Lee, S., Edwards, C. A., Arancon, N. Q., \& Metzger, J. D. (2002). The influence of humic acids derived from earthworm-processed organic wastes on plant growth. Bioresource technology, 84(1), 7-14.

Atiyeh, R. M., Subler, S., Edwards, C. A., Bachman, G., Metzger, J. D., \& Shuster, W. (2000). Effects of vermicomposts and composts on plant growth in horticultural container media and soil. Pedobiologia, 44(5), $579-590$.

Arduç, S. (2016). Yozgat koşullarında iki ekmeklik buğday (Triticum aestivum L.) çeşidinde hümik-fulvik asit uygulamasinın tane verimi ve bazl kalite özelliklerine etkisi (Doctoral dissertation, Fen Bilimleri Enstitüsü).

Akınc1, Ş. (2011). Hümik asitler, bitki büyümesi ve besleyici alımı. Marmara Fen Bilimleri Dergisi, 23(1), 46-56.

Canellas, L. P., Balmori, D. M., Médici, L. O., Aguiar, N. O., Campostrini, E., Rosa, R. C., ... \& Olivares, F. L. (2013). A combination of humic substances and Herbaspirillum seropedicae inoculation enhances the growth of maize (Zea mays L.). Plant and soil, 366(1), 119-132.

Canellas, L. P., Olivares, F. L., Aguiar, N. O., Jones, D. L., Nebbioso, A., Mazzei, P., \& Piccolo, A. (2015). Humic and fulvic acids as biostimulants in horticulture. Scientia horticulturae, 196, 15-27. https://doi.org/10.1016/j.scienta.2015.09.013

Dobbss, L. B., Pasqualoto Canellas, L., Lopes Olivares, F., Oliveira Aguiar, N., Peres, L. E. P., Azevedo, M., ... \& Facanha, A. R. (2010). Bioactivity of chemically transformed humic matter from vermicompost on plant root growth. Journal of Agricultural and Food Chemistry, 58(6), 3681-3688. https://doi.org/10.1021/jf904385c

Demirel, M. (2018). Farklı dozlarda katı biyogaz atıklarının buğday (Triticum aestivum L.) bitkisinin verim ve kalitesi üzerine etkisi (Master's thesis, Fen Bilimleri Enstitüsü).

Erdal, İ., Küçükyumuk, Z., TAPLAMACIOĞLU, D., \& Toftar, B. (2014). Kireçli bir toprakta humik ve fulvik asit uygulamalarının domatesin gelişimi ve beslenmesine etkileri. Toprak Bilimi ve Bitki Besleme Dergisi, 2(2), 70-74.

Halpern, M., Bar-Tal, A., Ofek, M., Minz, D., Muller, T., \& Yermiyahu, U. (2015). The use of biostimulants for enhancing nutrient uptake. Advances in agronomy, 130, 141-174. https://doi.org/10.1016/bs.agron.2014.10.001

Hernandez, O. L., Calderín, A., Huelva, R., Martínez-Balmori, D., Guridi, F., Aguiar, N. O., ... \& Canellas, L. P. (2015). Humic substances from vermicompost enhance urban lettuce production. Agronomy for sustainable development, 35(1), 225-232. https://doi10.1007/s13593014-0221-X

Karademir. S. (2019). Farkl Oranlarda Vermikompost Uygulamalarının Marulda (Lactuca Sativa L.) Bitki Gelişimi, Kalite Özellikleri Ve Besin Elementi İçeriği Üzerine Etkilerinin Belirlenmesi. YL Tezi. Bolu.

López, M. A. H., Ulery, A. L., Samani, Z., Picchioni, G., \& Flynn, R. P. (2011). Response of chile pepper (Capsicum annuum L.) to salt stress and organic and inorganic nitrogen sources: I. growth and yield. Tropical and Subtropical Agroecosystems, 14(1), 137-147.

Okur, N., Kayıkçığlu, H., Tunç, G. and Tüzel, Y. (2007). Organik tarımda kullanılan bazı organik gübrelerin topraktaki mikrobiyal aktivite üzerine etkisi. Ege Üniversitesi Ziraat Fakültesi Dergisi, 44(2), 65-80.

R. J. A., Senesi, N. and Wilkinson, K.J. (2008). Biophysical Chemistry of Fractal Structures and Processes in Environmental Systems. A John Wiley \& Sons, Ltd., West Sussex, 221-236.

Siebielec, G., Siebielec, S., \& Lipski, D. (2018). Long-term impact of sewage sludge, digestate and mineral fertilizers on plant yield and soil biological activity. Journal of Cleaner Production, 187, 372-379.

Taban, S., Turan, M. A., \& Katkat, A. V. (2013). Tarımda organik madde ve tavuk gübresi. Tavukçuluk Araştırma Dergisi, 10(1), 9-13.

Ünver, İ. T. D., \& Özbek, K. Y. (2011). Hiper toplayıı bitkilerle kadmiyumlu toprakların iyileştirilmesi ve gübre, humik asit ve şelat uygulamalarının etkinliği (Doctoral dissertation, Ankara Üniversitesi Fen Bilimleri Enstitüsü Toprak Bilimi ve Bitki Besleme Anabilim Dalı). 